\title{
Strenges 24/7-Alkoholabstinenzprogramm für Straffällige verringert die Gesamtmortalität
}

Fragestellung: Führt die Einführung eines streng kontrollierten Alkoholabstinenzprogramms für Straffällige zu einer Senkung der Mortalität der Bevölkerung?

Hintergrund: Seit 2003 wurde im US-Bundesstaat South Dakota schrittweise ein Alkoholabstinenzprogramm für gesetzlich auffällig gewordene Personen eingeführt. Organisiert über die Stadtverwaltungen wurden Betroffene verpflichtet, morgens und abends einen Atemalkoholtest durchzuführen. Wenn Alkohol nachgewiesen oder der Test verweigert oder abgebrochen wurde, erfolgte eine sofortige Inhaftierung für meist ein bis zwei Tage, ohne dass hierzu ein Gerichtsbeschluss notwendig war. Dieses Programm reduzierte Trunkenheitsfahrten um $12 \%$, häusliche Gewalt um $9 \%$ und Verkehrsunfälle junger Männer um 4\% [1].

Nicosia N, Kilmer B, Heaton P. Can a criminal justice alcohol abstention programme with swift, certain, and modest sanctions (24/7 Sobriety) reduce population mortality? A retrospective observational study. Lancet Psychiatry 2016; 3: 226-32
Patienten und Methodik: Von 2005 bis 2011 nahmen 16.932 Personen (3\% der Bevölkerung von South Dakota) durchschnittlich 122 Tage an diesem Programm teil. Etwa $80 \%$ der Teilnehmer hatten durch Trunkenheitsfahrten (64\% mehrfach, teils in Kom- bination mit anderen Straftaten) und 13\% durch Körperverletzungen gegen das Gesetz verstoßen. Mehr als $99 \%$ der Atemalkoholtests waren negativ, nur $0,36 \%$ der Tests waren im Durchschnitt nach 60 Tagen erstmals positiv. Die Mortalität wurde differenziert nach Ursachen erhoben für Herz-KreislaufErkrankungen, externe Verletzungen, gastrointestinale Erkrankungen und Krebs. Krebserkrankungen dienten als (kurzfristig) nicht durch Alkohol beeinflussbare Vergleichsgruppe. Verglichen wurde jeweils die Monatsmortalität vor der Einführung des Programms und danach. Zudem ging eine Reihe von potenziellen Einflussfaktoren in die Analyse mit ein.

Ergebnisse: Nach Einführung des 24/7-Abstinenzprogramms sank die Mortalität der Gesamtbevölkerung signifikant um $4,2 \%$ ( $<<0,01$, Konfidenzintervall 1,5-6,9\%). Die Mortalität durch externe Verletzungen sank um $11 \%(\mathrm{p}<0,1)$ und durch Herz-Kreislauf Erkrankungen um $7 \%(\mathrm{p}<0,05)$, während die Mortalität durch Krebserkrankungen nur um 0,4\% abnahm. Überdurchschnittlich sank die Mortalität bei Frauen (8\%) und bei Personen über 40 Jahren $(4,4 \%)$, obwohl diese Gruppen jeweils weniger als $25 \%$ der Stichprobe aufwiesen.

Schlussfolgerung: Die Senkung der Mortalität wird als kausale Folge des 24/7-Abstinenzprogramms interpretiert.

\section{- Kommentar von Derik Hermann, Mannheim}

\section{Alkohol tötet, Abstinenz verlängert Leben - eigentlich schon lange klar!}

Die gesundheitlichen Gefahren von Alkohol werden im alkoholpermissiven Deutschland kaum wahrgenommen. Alkohol führt zu etwa 40.000 Todesfällen pro Jahr, weitere 30.000 Menschen sterben an der Kombination von Alkohol und Tabak. Hinzu kommen etwa 50.000 unter Alkoholeinfluss begangene Gewalttaten pro Jahr, sowie 260 Tote und 16.856 Verletze durch Alkohol im Straßenverkehr. Evidenzbasierte Empfehlungen der WHO [2], den Alkoholkonsum durch politische Maßnahmen zu reduzieren, werden in Deutschland nur halbherzig umgesetzt.

Das Programm aus den USA zeigt, dass es auch anders geht. Eine ein- bis zweitägige Haftstrafe bei Verstoß gegen eine Abstinenzauflage ist zwar eine drastische, aber wirksame aversive Maßnahme zur Abstinenzsicherung. In verhaltenstherapeutischen Termini handelt es sich um eine „Bestrafung" von Alkoholkonsum mit unmittelbaren negativen Konsequenzen ähnlich wie bei dem alkoholaversiven Medikament Disulfiram, das ebenfalls erfolgreich die Abstinenz verlängern kann. Die engmaschigen Atemalkoholkontrollen und die sofortige Umsetzung der kurzen Haftstrafe verhindern Ausweichversuche und Gedanken, dass Alkoholkonsum unentdeckt bleiben könnte oder negative Folgen erst in entfernter Zukunft auftre- ten. Obwohl es sich um eine retrospektive Beobachtungsstudie mit niedriger Evidenzstufe handelt, ist die praktische Relevanz hoch. Aufgrund der positiven Effekte sollte erwogen werden, hierzulande ähnliche Programme für unter Alkoholeinfluss straffällig gewordene Personen einzuführen.
Referenzen
1. Kilmer B et al. Am J Public Health 2013; 103: e37-43
2. WHO 2010. http://www.who.int/substance_abuse/activities/gsrhua/en/ Last accessed June 2016

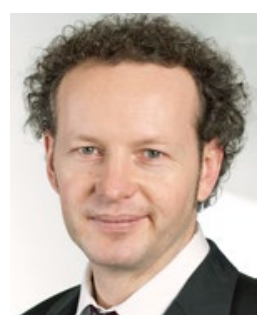

Prof. (apl.) Dr. Derik Hermann, Mannheim

Klinik für Abhängiges Verhalten und Suchtmedizin, Zentralinstitut für Seelische Gesundheit

E-Mail: derik.hermann@zi-mannheim.de 